\title{
The Correlation Between the Clinical Presentation and the Radiological Appearance in Patients with Myelitis at Alshaab Teaching Hospital-Sudan
}

\author{
Etedal Ahmed Ibrahim ${ }^{1}$, Hiba Hassan Ibrahim², Mohammed Adam EInour ${ }^{3}$, \\ Hyder Osman Mirghani ${ }^{3}$, Mohamed Nageeb Abdallah ${ }^{4}$ \\ ${ }^{1}$ Department of Internal Medicine, Medical College, Elneelain University, Khartoum, Sudan \\ ${ }^{2}$ Department of Internal Medicine, Medical College, Almughtaribeen University, Khartoum, Sudan \\ ${ }^{3}$ Department of Internal Medicine, Medical College, University of Tabuk, Tabuk, Kingdom of Saudi Arabia \\ ${ }^{4}$ Department of Internal Medicine, Faculty of Medicine, University of Khartoum, Khartoum, Sudan \\ Email address: \\ mohammedadam1@hotmail.com (M. A. Elnour)
}

\section{To cite this article:}

Etedal Ahmed Ibrahim, Hiba Hassan Ibrahim, Mohammed Adam Elnour, Hyder Osman Mirghani, Mohamed Nageeb Abdallah. The Correlation Between the Clinical Presentation and the Radiological Appearance in Patients with Myelitis at Alshaab Teaching HospitalSudan. American Journal of Internal Medicine. Vol. 5, No. 1, 2017, pp. 7-11. doi: 10.11648/j.ajim.20170501.12

Received: November 30, 2016; Accepted: December 28, 2016; Published: February 1, 2017

\begin{abstract}
Acute transverse myelitis is the leading cause of non-compressive myelopathy, linking the clinical presentation with Magnetic Resonance Imaging (MRI) may aid in proper stratification of patients improving management, we aimed to study the correlation between the clinical presentation of acute transverse myelitis with MRI findings. This cohort, hospitalbased study conducted at Alshaab Teaching Hospital, Sudan among patients with acute transverse myelitis, Participants signed a written informed consent then the clinical presentation was recorded and followed including symmetry and extent of the lesion, site of lesion, sensory involvement. Correlation between various clinical presentations and MRI findings was done. The ethical committee of Alshaab Teaching Hospital approved the research, and the Statistical Package for Social Sciences was used for data analysis. Out of 100 patients infections was observed in $25 \%$, and autoimmunity in $8 \%$, involvement of more than three segments of the spinal cord was commoner among patients with symmetrical and complete presentation, no significant difference was evident between patients with symmetrical and complete presentation, compared to partial asymmetrical presentation regarding the site of spinal cord involvement on MRI. Complete and symmetrical presentation are commonly associated with more than three segments involvement of the spinal cord, no significant difference was found between complete symmetrical presentation and partial asymmetrical presentation regarding the site of involvement on MRI.
\end{abstract}

Keywords: Transverse Myelitis, Radiological Finding, Sudan

\section{Introduction}

Acute transverse myelitis [ATM] has been reported as the leading cause of non-compressive spinal cord pathologies [1]. Transverse myelitis is an inflammatory process across the spinal cord that can damage the myelin, and a scar may develop resulting in loss of communication between the spinal cord and the rest of the body [2].

The term acute transverse myelitis denotes the presence of inflammation ruling out non-inflammatory causes; possible causes include infections, auto-immune disorders, inflammatory, vascular, and neoplastic and metastatic disorders [3]. ATM can occur as an isolated inflammatory disease, or as part of multifocal CNS demyelinating diseases such as multiple sclerosis (MS), Neuromyelitis Optica (NMO) acute disseminated encephalomyelitides (ADEM), or it can also be associated with systemic rheumatological disorders. Some remain with no apparent cause after ruling all possible causes on follow up and is referred to as idiopathic [4].

The clinical picture of transverse myelitis consist of ascending pattern of weakness and sensory dysfunctions involving the lower extremities; the upper limbs are not usually involved, abdominal or back pain may be associated, fever when present usually but not always denote infections $[5]$.

Acute transverse myelitis (ATM), a subgroup of different medical conditions that lead to transverse spinal cord 
syndromes, it could be idiopathic or inflammatory spinal cord disease. Idiopathic. ATM is diagnosed based on the following criteria: bilateral symptoms and signs attributable to spinal cord disease, the presence of focal cord inflammation by magnetic resonance imaging and cerebrospinal fluid studies, and the exclusion of many diseases, especially cord compression and "diseaseassociated ATM," as Sjögren disease. The presentation with sensory and autonomic dysfunctions with acute or subacute onset, progressing to the maximum neurological deficit in $4 \mathrm{~h}$ to 21 days, most of the cases improve in full or partial [6].

Radiology is of paramount importance to rule out spinal cord compression, and is one of exclusion criteria to diagnose idiopathic transverse myelitis; other exclusion criteria are Evidence of thrombosis of the anterior spinal artery, connective tissue disease, infection, optic neuritis, and multiple sclerosis [7].

The epidemiology of complete idiopathic transverse myelitis is uncertain and depends on the underlying pathology, previous studies in the United States of America, Europe and Israel ranged from 1-4 cases per million. The disease has a bimodal distribution of age presentation peaking at ages 10-19 and 30-39 years [8] [9].

Acute transverse myelitis is the leading cause of noncompressive myelopathy, linking the clinical presentation with Magnetic Resonance Imaging (MRI) may aid in proper stratification of patients improving management, No researchers have studied the association of the clinical features of acute transverse myelitis to the radiological picture in Sudan. Thus we conducted this survey, in the present study, we aimed to explore the correlation between the clinical presentation of acute transverse myelitis with MRI findings.

\section{Material \& Methods}

This cohort hospital-based study conducted in Alshaab Teaching Hospital, Sudan during the period from April to December 2012. One hundred patients admitted with bilateral sensory, motor, and autonomic neurological dysfunction attributed to the spinal cord with or without optic neuritis and higher function involvement were approached, those with unilateral symptoms and symptoms of sudden onset were excluded as were those with spinal cord compression. Participants were asked to sign a written informed consent then interviewed by the researchers. A structured questionnaire was used, information collected include: demographic data, onset and mode of clinical presentation, past medical history, family history, Spinal Magnetic Resonance Imaging ( MRI) with a magnet strength of 1.5 Tesla to assess the radiological appearance including both axial and sagittal planes with $\mathrm{T} 1$, and $\mathrm{T} 2$-weighted sequences, brain MRI in selected cases with axial, sagittal, and coronal with T1, and T2-weighted and FLAIR images, and the initial response to treatment.

ATM is diagnosed based on the following criteria:

- Bilateral symptoms and signs attributable to spinal cord disease.

- The presence of focal cord inflammation by magnetic resonance imaging and cerebrospinal fluid studies.
- Exclusion of other diseases, especially cord compression and "disease-associated ATM," as Sjögren disease.

- The presentation with sensory and autonomic dysfunctions with acute or subacute onset, progressing to the maximum neurological deficit in $4 \mathrm{~h}$ to 21 days, most of the cases improve in full or partial. The treatment is symptomatic and a corticosteroid to halt the inflammatory process. The diagnosis of specific inflammatory and autoimmune myelopathies (paraneoplastic myelitis and neuromyelitis optica spectrum diseases) is crucial to establish therapeutic strategies, and now possible with the use of specific biomarkers [6]

The ethical committee of Alshaab Teaching Hospital approved the research and the Statistical Package for Social Sciences version 21 was used for data analysis. The mean, standard deviation and frequencies were defined. The chisquare test was used to compare categorical variables, and Pvalue of $<0.05$ considered significant.

\section{Results}

Out of 100 patients with acute transverse myelitis 59\% were females, their ages ranged from 15-75 with a mean of $39.17 \pm 12.78$ years, antecedent illness was present in $25 \%$, the majority $[70 \%]$ had an acute presentation. The clinical presentation peaked in 1-6 days in 39\% of patients, and in 14 weeks in $47 \%$ of patients, more than two-thirds [75\%] presented with sensory symptoms, followed by lower limb weakness [68\%], and sphincteric disturbances in [66\%]. The pattern of progression was ascending in the majority of patients [66\%], followed by the circumferential [27\%], while ascending pattern was detected in [11\%]. More than one-fifth of patients [21\%] had significant past neurological history. The commonest underlying cause was infection [25\%], followed by autoimmune disorders in [8\%] Table (1). Hyperintense lesion in the spinal MRI was the commonest abnormality and detected in [75\%] of patients, the most common region affected was the dorsal segments of the spinal cord [49\%], followed by the cervical [8\%]. Table [2] depicted other spinal MRI characteristics.

In the current data hyperintense lesion involving $>2 / 3$ of the spinal cord was commoner incomplete, and symmetrical presentation as compared to partial and asymmetrical [32\% vs. $24 \%$, and $30 \%$ vs. $26 \%$ respectively with on significant statistical difference Table [3]. The dorsal column was affected more incomplete and symmetrical involvement [34\%vs. $15 \%$, and $39 \%$ vs. $10 \%$ respectively with significant statistical difference Table [4]. Table No [5] illustrated the length of spinal cord involved in correlation to pattern of clinical presentation in which; more than 3 segments of the spinal cord were involved in $29 \%$ in complete involvement and $26 \%$ in partial with no significant statistical difference, while it was present in $28 \%$, and $27 \%$ in symmetrical and asymmetrical involvement respectively. In the minority of patients [15\%] in whom the MRI brain was done it was normal, more than two-thirds of patients improved with methylprednisolone [64\%], and no improvement was observed in [35\%]. 
Table 1. Basic clinical characteristics of the study group.

\begin{tabular}{ll}
\hline Character & $\mathbf{\%}$ \\
\hline Males & 41 \\
Females & 59 \\
Antecedent febrile illness & 25 \\
Onset of clinical features & 70 \\
Acute & 27 \\
Sub-acute & 3 \\
Chronic & \\
Peak of clinical features & 39 \\
$1-6$ days & 47 \\
$1-4$ weeks & 14 \\
More than four weeks & \\
Symptoms & 57 \\
Backache & 68 \\
Lower limb weakness & 32 \\
Four limb weakness & 75 \\
Sensory involvement & 66 \\
Sphincteric disturbances & \\
Pattern of progression & 62 \\
Ascending & 11 \\
Descending & 27 \\
Circumferential & 21 \\
Past medical history & \\
Causes & 8 \\
Autoimmune disease & 25 \\
post infectious & \\
\hline
\end{tabular}

Table 2. MRI characteristics of the study group.

\begin{tabular}{|c|c|c|c|c|}
\hline \multicolumn{3}{|l|}{ Character } & \multicolumn{2}{|l|}{ No \% } \\
\hline \multicolumn{5}{|c|}{ Abnormal scan according to time interval } \\
\hline \multicolumn{3}{|c|}{ Less than five days } & \multicolumn{2}{|l|}{$12(12 \%)$} \\
\hline \multicolumn{3}{|l|}{ More than five days } & \multicolumn{2}{|l|}{$73(73 \%)$} \\
\hline \multicolumn{5}{|l|}{ Radiological appearance } \\
\hline \multicolumn{3}{|l|}{ Cord swelling } & \multicolumn{2}{|l|}{$10(10 \%)$} \\
\hline \multicolumn{3}{|l|}{ Hyperintense $>2 / 3$ of cord } & \multicolumn{2}{|l|}{$56(56 \%)$} \\
\hline \multicolumn{3}{|l|}{ Hyperintense $>1 / 2$ cord } & \multicolumn{2}{|l|}{$19(19 \%)$} \\
\hline \multicolumn{3}{|l|}{ Normal finding } & \multicolumn{2}{|l|}{$15(15 \%)$} \\
\hline \multicolumn{5}{|l|}{ Site of the lesion } \\
\hline \multicolumn{3}{|l|}{ Dorsal } & \multicolumn{2}{|l|}{$49(49 \%)$} \\
\hline \multicolumn{3}{|l|}{ Cervicodorsal } & \multicolumn{2}{|l|}{$21(21 \%)$} \\
\hline \multicolumn{3}{|l|}{ Cervical } & \multicolumn{2}{|l|}{$8(8 \%)$} \\
\hline \multicolumn{3}{|l|}{ Whole spinal cord } & \multicolumn{2}{|l|}{$7(7 \%)$} \\
\hline \multicolumn{3}{|l|}{ Normal } & \multicolumn{2}{|l|}{$15(15 \%)$} \\
\hline \multicolumn{5}{|c|}{ Length of vertebral segments involved } \\
\hline \multicolumn{3}{|l|}{$<3$ segments } & \multicolumn{2}{|l|}{$30(30 \%)$} \\
\hline \multicolumn{3}{|l|}{$>3$ segments } & \multicolumn{2}{|l|}{$55(55 \%)$} \\
\hline \multicolumn{3}{|l|}{ Normal scan } & \multicolumn{2}{|l|}{$15(15 \%)$} \\
\hline Character & Normal \% & Hyper intensity $>2 / 3$ of cord & Hyper intensity $<1 / 2$ of cord & Cord swelling \\
\hline Cord Dysfunction & & & & \\
\hline Complete & 9 & 32 & 9 & 7 \\
\hline $\begin{array}{l}\text { partial } \\
\text { the symmetry of signs }\end{array}$ & 6 & 24 & 10 & 3 \\
\hline Symmetrical & 12 & 30 & 12 & 12 \\
\hline Asymmetrical & 3 & 26 & 7 & 7 \\
\hline
\end{tabular}

Table 4. correlation between the pattern of spinal cord dysfunction and the site of the lesion on MRI.

\begin{tabular}{llllll}
\hline Character & Normal \% & Cervical \% & Dorsal \% & Cervico-dorsal \% & Whole cord \% \\
\hline Cord Dysfunction & & & & & \\
Complete & 9 & 0 & 34 & 9 & 5 \\
Partial & 5 & 8 & 15 & 12 & 2 \\
Degree of symmetry & 12 & 1 & 39 & 7 & 1 \\
Symmetrical & 3 & 7 & 10 & 14 & 6 \\
Asymmetrical & & & & & \\
\hline
\end{tabular}


Table 5. correlation between the pattern and degree of spinal cord dysfunction and length of spinal segments involved.

\begin{tabular}{llll}
\hline character & Normal scan \% & $<\mathbf{3}$ segments involved \% & $>\mathbf{3}$ segments involved \% \\
\hline Cord Dysfunction & & & 29 \\
Complete & 9 & 11 & 26 \\
partial & 6 & & 28 \\
Degree of symmetry & & 20 & 27 \\
Symmetrical & 12 & 10 & 27 \\
Asymmetrical & 3 & & 28 \\
\hline
\end{tabular}

Table 6. Brain MRI of the study group.

\begin{tabular}{ll}
\hline MRI & \% \\
\hline Normal & 15 \\
abnormal & 0 \\
Not done & 85 \\
\hline
\end{tabular}

Table 7. Response to therapy among the study group.

\begin{tabular}{ll}
\hline Response to treatment & \% \\
\hline Improvement & 64 \\
No improvement & 35 \\
Treatment not given & 1 \\
\hline
\end{tabular}

\section{Discussion}

Acute transverse myelitis, a fragment of disseminated vasculomyelinopathy is pathogenetically similar to acute disseminated encephalomyelitis. The hallmark acute transverse myelitis is perivenular inflammation and surrounding demyelination MRI is the investigation of choice investigation to detect such lesions in the spinal cord. MRI imaging may be normal in early stages of the disease, and no signal intensity is detected, later as the illness progress local enlargement of the spinal cord and increased signal intensity on long repetition time/echo time sequences are shown [10].

In the present study, the majority of patients had their MRI done more than five days from the symptoms onset, with an abnormality detected in $85 \%$, similar to Prabhakar study [11]. In the current study high signal intensity lesions occupying $>2 / 3$ of the spinal cord was evident in $56 \%$ of patients by Murthy et al. [12] in India, who concluded similar findings $[61.5 \%]$.

The present data found that the dorsal spines were involved in the majority of MRI [49\%], by previous researchers who concluded similar results [11]. Previous researchers found similar results [3]

In contradiction to then present finding De Seze et al. [14] found that the majority of lesions were in the cervical segments. In the current research involvement of $>3$ segments of the spinal cord was observed in $55 \%$ of patients in similarity with Choi et al. [15] who reported that $>3$ segment involvement was found in $54 \%$ of patients with transverse myelitis, particularly those with Neuromyelitis Optica.

Regarding the pattern of presentation, the complete and symmetrical presentations were commoner in patients with hyperintense areas involving more than two-thirds of the spinal cord, while partial, and a symmetrical presentations were commoner among hyperintense MRI lesions involving $<1 / 2$ of the spinal cord similar to previous literature [11, 15].

In the present study, no statistically significant difference was evident between the clinical presentation and the site of the lesion; one plausible explanation is that the presence of spinal lesions involving multiple levels can lead to versatility in clinical features [16].

\section{Conclusion}

Infections was detected in $25 \%$, of the study group, involvement of more than three segments of the spinal cord was commoner among patients with symmetrical and complete presentation, no significant difference was evident between patients with symmetrical and complete presentation, compared to partial asymmetrical presentation regarding the site of spinal cord involvement on MRI. Complete and symmetrical presentation are commonly associated with more than three segments involvement of the spinal cord, no significant difference was found between complete symmetrical presentation and partial asymmetrical presentation regarding the site of involvement on MRI.

Limitations of the study are the small size of the survey group, and the study was conducted at a single tertiary center so generalization cannot be insured, further larger multicenter studies are needed.

\section{References}

[1] Chaurasia RN, Verma N, Joshi D, Misra S. Etiological spectrum of non-traumatic myelopathies: Experience from a tertiary care centre. J Assoc Physics India 2006; 54: 445-8.

[2] De seze J, Lanctin C, Lebrun $\mathrm{C}$ et al. Idiopathic acute transverse myelitis: application of the recent diagnostic criteria. Neurology 2005; 65: 1950-3.

[3] Alper G Petropoulou KA Fitz CR Kim Y. IDIOPATHIC ACUTE TRANSVERSE MYELITIS IN CHILDREN: An analysis and discussion of MRI findings. Mult Scler. 2011 Jan; 17 (1): 74-80.

[4] Transverse Myelitis Consortium Working group Proposed diagnostic criteria and nosology of acute transverse myelitis. Neurology. 2002; 59: 499-505.

[5] West, TW. "Transverse myelitis - a review of the presentation, diagnosis, and initial management." Discovery Medicine 2013; 16 (88): 167-177.

[6] Sá MJ. Acute transverse myelitis: a practical reappraisal. Autoimmun Rev. 2009 Dec; 9 (2): 128-31. doi: 10.1016/j.autrev.2009.04.005. Epub 2009 Apr 21.

[7] "Proposed diagnostic criteria and nosology of acute transverse myelitis.". Neurology (Transverse Myelitis Consortium Working Group) 59 (4): 499-505.27 August 2002.

[8] Klein NP, Ray P, Carpenter D, Hansen J, Lewis E, Fireman B, Black S, Galindo C, Schmidt J, Baxter R. Rates of autoimmune diseases in Kaiser Permanente for use in vaccine adverse event safety studies. Vaccine. 2010 Jan 22; 28 (4): 1062-8. doi: 10.1016/j.vaccine.2009.10.115. Epub 2009 Nov 5. 
[9] Mealy MA, Wingerchuk DM, Greenberg BM, Levy M. Epidemiology of neuromyelitis optica in the United States: a multicenter analysis. Arch Neurol. 2012 Sep; 69 (9): 1176-80. doi: 10.1001/archneurol.2012.314.

[10] Murthy JM, Reddy JJ, Meena AK, Kaul S. Acute transverse myelitis: MR characteristics 1999; 47 (4): 290-93.

[11] Prabhakar S, Syal P, Singh P, Lal V, Khandelwal N, Das CP. Non-compressive myelopathy: clinical and radiological study. Neurol India 1999; 47: 294-9.

[12] Murthy JM, Reddy JJ, Meena AK, Kaul S. Acute transverse myelitis: MRI characteristics: Neurol India 1999;47:290.
[13] De Seze J, Lanctin C, Lebrun C et al. Idiopathic acute transverse myelitis: application of the recent diagnostic criteria. Neurology 2005; 65: 1950-3.

[14] Choi KH, Kee KS, Chung SO et al. Idiopathic transverse myelitis: MR characteristics. Am J Neuroradiol 1996; 17: 1151-1160.

[15] Goh C, Phal PM, Desmond PM. Neuroimaging in acute transverse myelitis. Neuroimaging Clin N Am. 2011 Nov; 21 (4): 951-73, x. doi: 10.1016/j.nic.2011.07.010. 